\title{
The Generalized Riemann Problem for Chaplygin Gas with Combustion*
}

\section{Yujin Liu}

School of Mathematics and Statistics, Shandong University of Technology, Zibo, China

Email: yjliu98@126.com

How to cite this paper: Liu, Y.J. (2019) The Generalized Riemann Problem for Chaplygin Gas with Combustion. Journal of Applied Mathematics and Physics, 7, 2738-2750. https://doi.org/10.4236/jamp.2019.711187

Received: October 11, 2019

Accepted: November 5, 2019

Published: November 8, 2019

Copyright (c) 2019 by author(s) and Scientific Research Publishing Inc. This work is licensed under the Creative Commons Attribution International License (CC BY 4.0).

http://creativecommons.org/licenses/by/4.0/

\begin{abstract}
We study the generalized Riemann problem of the Chapman-Jouguet model for an ideal combustible Chaplygin gas. By analyzing the wave curves in the phase plane, we construct uniquely the solution of the generalized Riemann problem under the global entropy conditions. We find that although there is no combustion wave of the corresponding Riemann solution, the combustion wave may occur after perturbation which reveals the instability of the unburnt gas.
\end{abstract}

\section{Keywords}

Generalized Riemann Problem, Chaplygin Gas, Detonation Wave,

Deflagration Wave, Global Entropy Conditions

\section{Introduction}

In the present paper we study the one-dimensional adiabatic, inviscid flow of ideal combustible Chaplygin gas in Euler coordinates

$$
\left\{\begin{array}{l}
\rho_{t}+(\rho u)_{x}=0, \\
(\rho u)_{t}+\left(\rho u^{2}+p\right)_{x}=0, \\
(\rho E)_{t}+(\rho u E+p u)_{x}=0, \\
q(x, t)= \begin{cases}0 & \text { if } \sup _{0 \leq y \leq t} T(x, y)>T_{i} \\
q(x, 0) & \text { otherwise, }\end{cases}
\end{array}\right.
$$

where $\rho, u, p<0, T$ and $T_{i}$ are respectively the density, velocity, pressure, temperature and ignition temperature. $q$ is the chemical binding energy. In addition 
to the kinetic energy $\frac{u^{2}}{2}$ and internal energy $e$, the total energy $E$ also contains the chemical binding energy $q$, i.e., $E=\frac{u^{2}}{2}+e+q$. For simplicity, the state equation is $p=-\frac{1}{\rho}$ and the internal energy $e=-\frac{p}{2 \rho}$. We also assume that the combustion process is exothermic, i.e., the energy used up in recombing the atoms to form the new molecules is smaller for the burnt gas than the binding energy of the unburnt gas [1]. Chaplygin gas was introduced by Chaplygin [2] and it arose many authors' interest [3] [4] [5] [6].

There are two well-known mathematical models, which we use to study combustion phenomena in chemically reactive gas: the Chapman-Jouguet (CJ) model and the Zeldovich-von Neumann-Döring (ZND) model [1] [7]. CJ model has infinite reaction velocity, and ZND model has finite reaction velocity, which exists finite reaction process. Courant and Friedrichs [1] studied the CJ combustion model and obtained its partial solutions including deflagrations and detonations. Zhang and Zheng [8] studied the above CJ model further in Lagrangian coordinates

$$
\left\{\begin{array}{l}
u_{t}+p_{x}=0, \\
\tau_{t}-u_{x}=0, \\
E_{t}+(u p)_{x}=0, \\
q(x, t)= \begin{cases}0 & \text { if } \sup _{0 \leq y \leq t} T(x, y)>T_{i} \\
q(x, 0) & \text { otherwise }\end{cases}
\end{array}\right.
$$

where $\tau$ is the specific volume which satisfies that $\tau=\frac{1}{\rho}$, here $p>0$ and $E=\frac{u^{2}}{2}+\frac{p \tau}{\gamma-1}+q$ for polytropic gases, $\gamma>1$ is the adiabatic exponent. And they obtained constructively the existence and uniqueness of the Riemann solution under the global entropy conditions. Liu and Sheng [9] modified the global entropy conditions in [8] and constructed uniquely the generalized Riemann solutions of the CJ model (2).

Besides the above CJ combustion model, another important model is the ZND combustion model. Tan and Zhang [10] studied the Riemann problem for the selfsimilar ZND model

$$
\left\{\begin{array}{l}
u_{t}+p_{x}=0, \\
\tau_{t}-u_{x}=0, \\
E_{t}+(u p)_{x}=0, \\
q(x, t)=-\frac{k}{t} \varphi(T) q,
\end{array}\right.
$$

and proved that the selfsimilar solutions of the corresponding CJ model (2) are the limits of the selfsimilar solutions of the selfsimilar ZND model (3) as the reaction velocity $k \rightarrow \infty$ under the assumption that the temperatures along the 
SZND burning solutions are higher than $T_{i}$. Hsu and Lin [11] studied the two combustion models (2) and (3) further and they can determine when the temperatures along the burning solutions of the selfsimilar ZND model (3) are higher than $T_{i}$ and can determine when the selfsimilar solutions of the CJ model (2) are the limits of the self-similar solutions of the selfsimilar ZND model (3) when $k \rightarrow \infty$.

In [12], we studied the Riemann problem for (1) with the initial data

$$
(\tau, u, p, q)= \begin{cases}\left(\tau_{-}, u_{-}, p_{-}, q_{-}\right) & \text {if } x<0 \\ \left(\tau_{+}, u_{+}, p_{+}, q_{+}\right) & \text {if } x>0\end{cases}
$$

where

$$
q_{ \pm}= \begin{cases}0, & T_{ \pm}>T_{i}, \\ 0 \text { or } q_{0}, & T_{ \pm} \leq T_{i} .\end{cases}
$$

In this paper we study the generalized Riemann problem for (1) with the initial data

$$
(\tau, u, p, q)(x, 0)=\left(\hat{\tau}_{ \pm}, \hat{u}_{ \pm}, \hat{p}_{ \pm}, \hat{q}_{ \pm}\right)(x), \pm x>0,
$$

where $\hat{q}_{ \pm}(x)=q_{ \pm}, \hat{u}_{ \pm}(x)$ and $\hat{\tau}_{ \pm}(x), \hat{p}_{ \pm}(x)$ are arbitrary smooth functions satisfying

$$
\lim _{x \rightarrow 0 \pm}\left(\hat{\tau}_{ \pm}, \hat{u}_{ \pm}, \hat{p}_{ \pm}\right)(x)=\left(\tau_{ \pm}, u_{ \pm}, p_{ \pm}\right) .
$$

The problem (1) and (6) may be regarded as a perturbation of the corresponding Riemann problem (1) and (4). The generalized Riemann problem is the new initial value problem which has the new initial values (6). Naturally, we wonder if the solutions of (1) and (6) are similar to the corresponding Riemann solutions of (1) and (4) in the neighborhood of the origin. Our results show that for most of the cases, the structures of the Riemann solutions are able to retain their forms after perturbation while for some other cases; the perturbation brings essential changes to the structure of the Riemann solutions. We find that although there is no combustion wave of the corresponding Riemann solution, the combustion wave may occur after perturbation which reveals the unburnt gas is unstable. We assume that the combustion process takes place with an infinite rate of reaction, i.e., $q$ takes only two values: $q_{0}>0$ for the unburnt gas and 0 for the burnt gas.

This paper is organized as follows. In Section 2, we present some preliminaries. In Section 3, we construct the solutions of the generalized Riemann problem for the CJ combustion model (1) with the initial data (6) under the global entropy conditions. Our main result is given in Section 4.

\section{Preliminaries}

As a preparation, we study the Riemann problem for (1) and (4) and refer the detailed discussions to [12] [13].

There are two characteristic roots of (1), which are 


$$
\lambda=u \pm \sqrt{\frac{-p}{\rho}} \equiv \lambda_{ \pm}, \lambda=u \equiv \lambda_{0},
$$

system (1.1) is strictly hyperbolic. The corresponding right characteristic vector of $\lambda_{i}(i= \pm, 0)$ is

$$
\vec{r}_{ \pm}=\left(1, \pm \frac{1}{\rho} \sqrt{\frac{-p}{\rho}}, \frac{-p}{\rho}\right)^{\mathrm{T}}, \vec{r}_{0}=(1,0,0)^{\mathrm{T}} .
$$

Due to $\nabla \lambda_{i} \cdot \vec{r}_{i} \equiv 0, i=-,+, 0$, we know that all characteristic fields are linearly degenerate, where the symbol $i=-,+, 0$ corresponds respectively to the first, third and second characteristic field.

For a given left state $U_{l}\left(\rho_{l}, u_{l}, p_{l}\right)$, all the states $U(\rho, u, p)$ that can be connected on the right to $U_{l}$ by a forward or backward rarefaction wave curve $\vec{R}(l)(\overleftarrow{R}(l))$ are given as

$$
\left\{\begin{array}{l}
p \rho=p_{l} \rho_{l} \\
u=u_{l} \pm \frac{p-p_{l}}{\sqrt{-p_{l} \rho_{l}}}, \quad\left(p>p_{l} \text { or } p<p_{l}\right),
\end{array}\right.
$$

the forward or backward shock wave curve $\vec{S}(l)(\bar{S}(l))$

$$
\left\{\begin{array}{l}
p \rho=p_{l} \rho_{l}, \\
\frac{u-u_{l}}{p-p_{l}}= \pm \sqrt{-\frac{1}{p_{l} \rho_{l}}}, \quad\left(p_{l}>p \text { or } p_{l}<p\right),
\end{array}\right.
$$

the contact discontinuity $J$

$$
\left\{\begin{array}{l}
{[u]=[p]=0} \\
\rho_{l} \neq \rho_{r}
\end{array}\right.
$$

where $[u]=u_{r}-u_{l}$, etc.

Since the characteristic line from the initial point will overlap, from [14] we should consider the delta shock wave solution.

Assume that

$$
\begin{gathered}
\rho=\rho_{0}+\omega(t) \delta(x-x(t)), \quad \rho_{0}= \begin{cases}\rho_{l}, & x<x(t), \\
\rho_{r}, & x>x(t),\end{cases} \\
u(x, t)= \begin{cases}u_{l}, & x<x(t), \\
u_{\delta}, & x=x(t), \\
u_{r}, & x>x(t),\end{cases} \\
p(x, t)= \begin{cases}p_{l}, & x<x(t), \\
0, & x=x(t), \\
p_{r}, & x>x(t),\end{cases}
\end{gathered}
$$

where $\delta(x-x(t))$ is the delta measure supported on $x=x(t), \omega(t)$ denotes the weight of the delta shock wave $x=x(t)$, it follows that when $\rho_{r} \neq \rho_{l}$

$$
\omega(t)=\sqrt{\rho_{l} \rho_{r}\left(u_{r}-u_{l}\right)^{2}-\left(\rho_{r}-\rho_{l}\right)\left(p_{r}-p_{l}\right)} t
$$




$$
u_{\delta}=\frac{\rho_{r} u_{r}-\rho_{l} u_{l}+\frac{\mathrm{d} \omega(t)}{\mathrm{d} t}}{\rho_{r}-\rho_{l}},
$$

when $\rho_{r}=\rho_{l}$,

$$
\begin{gathered}
\omega(t)=\left(\rho_{l} u_{l}-\rho_{r} u_{r}\right) t \\
u_{\delta}=\frac{1}{2}\left(u_{r}+u_{l}\right) .
\end{gathered}
$$

Furthermore, the delta shock wave must satisfy the following entropy condition

$$
u_{r}+\sqrt{-\frac{p_{r}}{\rho_{r}}}<\frac{\mathrm{d} x(t)}{\mathrm{d} t}<u_{l}-\sqrt{-\frac{p_{l}}{\rho_{l}}} .
$$

The above solution is called the delta shock wave solution which is denoted by $S_{\delta}$.

In the phase plane $(u, p)$ (Figure 1), we can draw wave curves $\bar{R}(l), \bar{S}(l)$, $\vec{R}(l), \vec{S}(l)$ and the straight line $u=u_{l}+\frac{p+p_{l}}{\sqrt{-\rho_{l} p_{l}}}$ through the left state $(I)$ (Figure 1(a)). We know that if $\vec{S}(r)$ is located in the left side of the straight line, we obtain a delta shock wave solution. Similarly, we draw wave curves $\bar{R}(r), \bar{S}(r), \vec{R}(r), \vec{S}(r)$ and the straight line $u=u_{r}-\frac{p+p_{r}}{\sqrt{-\rho_{r} p_{r}}}$ through the left state $(r)$ (Figure $1(\mathrm{~b})$ ). If $\bar{S}(l)$ is located in the right side of the straight line, we get a delta shock wave solution.

Now we investigate the combustion wave curves in the $(u, p)$ plane. From the Rankine-Hugoniot relations

$$
\left\{\begin{array}{l}
\zeta[u]=[p] \\
\zeta[\tau]=-[u] \\
\zeta[E]=[u p]
\end{array}\right.
$$

we have

$$
-\tau_{r} p+p_{r} \tau=2 q_{0}>0,
$$

which is the Hugoniot curve in the $(\tau, p)$ plane. And we obtain the forward combustion wave curve which is connected to the right state $(r)$ in the $(u, p)$ plane (Figure 2)

$$
\vec{D}(r): \frac{u-u_{r}}{p-p_{r}}=\sqrt{-\frac{2 q_{0}+\tau_{r}\left(p-p_{r}\right)}{p_{r}\left(p-p_{r}\right)}},
$$

where $p_{r}<p<0$ or $p<p_{r}-\frac{2 q_{0}}{\tau_{r}}$. And $\vec{D}(r)$ loses meaning when $p_{r}-\frac{2 q_{0}}{\tau_{r}}<p<p_{r} . \vec{D}(r)$ is composed of two separate branches. Our claim is that when $p_{r}<p<0$ the combustion wave curve is detonation wave (DT) 


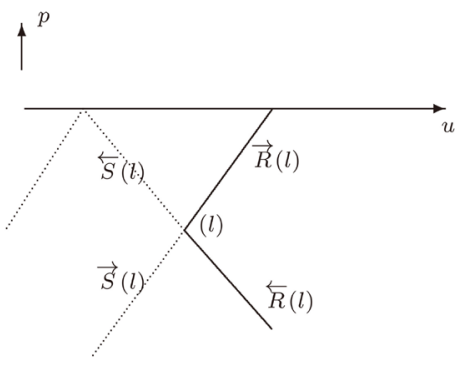

(a) The backward wave curves in (u, p).

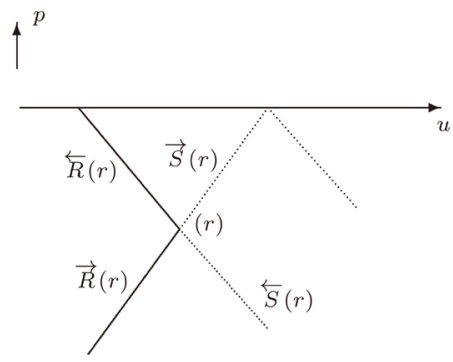

(b) The forward wave curves in $(u, p)$.

Figure 1. The non-combustion wave curves in $(u, p)$.

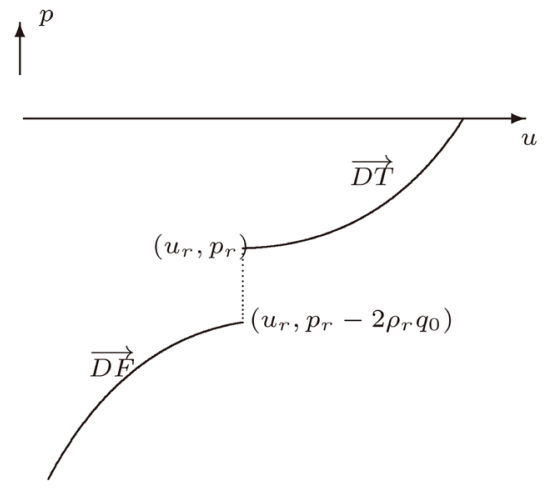

Figure 2. The combustion wave curve in $(u, p)$.

because along the detonation wave both pressure and density increase and along the deflagration wave both pressure and density decrease [1].

When $q_{+}>0$, the forward wave curve $\vec{W}(+)$ which can be linked to the state $(+)$ is given by

$$
\vec{W}(+):=\vec{W}_{S}(+) \cup S_{\delta}(+) \cup \overrightarrow{D F}(+) \cup \overrightarrow{D T}(+),
$$

where

$$
\vec{W}_{S}(+):=\vec{R}(+) \bigcup \vec{S}(+) .
$$

Similarly, we can construct the backward wave curve $\bar{W}(-)$ which can be linked to the state (-).

When $q_{+}=q_{-}=0$, the gas on both sides are burnt and there is no combustion wave. The Riemann problem is resolved in [13].

When $q_{+}$and $q_{-}$are not both zero, combustion wave will occur. For simplicity, we just need to consider the following two cases.

Case 2.1. $q_{-}=0, q_{+}=q_{0}>0$ and the gas on the left-hand side is burnt and on the right-hand side is unburnt.

For this case, the backward wave curve is $\bar{W}(-)=\bar{W}_{S}(-) \cup S_{\delta}(-)$, and the forward wave curve is $\vec{W}(+)=\vec{W}_{S}(+) \cup S_{\delta}(+) \cup \overrightarrow{D F}(+) \cup \overrightarrow{D T}(+)$ (Figure 3).

Our discussions are divided into two possibilities.

Subcase 2.1.1 When $u_{-}-u_{+}<\sqrt{-\frac{p_{-}}{\rho_{-}}}+\sqrt{-\frac{p_{+}}{\rho_{+}}}$. 


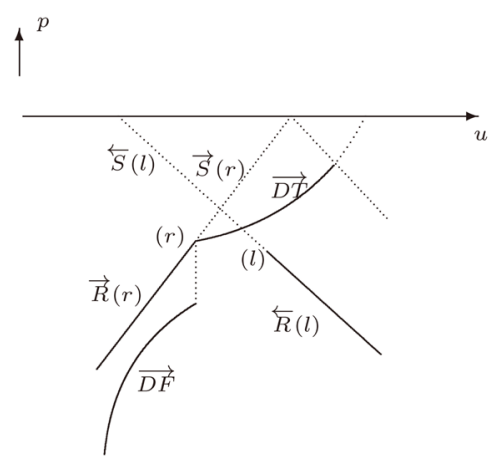

Figure 3. The wave curves in Case 2.1.

If the intersection point of $\bar{W}(-)$ and $\vec{W}(+)$ is unique, the Riemann solution is also unique; otherwise, we select the unique Riemann solution according to the following global entropy conditions:

We select the unique solution from the four intersection points (at most) of the forward wave curves connecting $(+)$ and the backward wave curves connecting (-) in the following order:

$A$. the solution with the parameter $\beta$ as small as possible, where $\beta$ is defined as oscillation frequency of $T(\xi)$ between the set $\left\{\xi \in R^{1}: T(\xi) \leq T_{i}\right\}$ and the set $\left\{\xi \in R^{1}: T(\xi)>T_{i}\right\}$;

$B$. The solution containing as many combustion wave as possible.

We denote the intersection point of $\bar{W}_{S}(-)$ and $\vec{W}_{S}(+)$ by $*_{S}$ and the intersection point of $\bar{W}_{S}(-)$ and $\overrightarrow{D F}(+)$ or $\overrightarrow{D F}(+)$ by $*_{D}$. We denote the temperature at the point $*_{S}, *_{D}$ on $\bar{W}_{S}(-)$ by $T_{S}, T_{D}$, respectively. The temperature at $*_{D}$ on $\overrightarrow{D F}(+)$ or $\overrightarrow{D F}(+)$ is greater than $T_{i}$ according to the assumption that the combustion process is exothermic (Figure 4).

1) When $T_{-}>T_{i}, T_{D}>T_{i}$, then $\beta\left(*_{S}\right)=1, \beta\left(*_{D}\right)=1$, from the condition $B$, we select $*_{D}$ and obtain a combustion wave solution containing a $D F$ or $D T$ (Figure 4(a)).

2) When $T_{-}>T_{i}, T_{D} \leq T_{i} \quad\left(\Rightarrow T_{S} \leq T_{i}\right)$, then $\beta\left(*_{S}\right)=1, \beta\left(*_{D}\right)=3$, from the condition $A$, we select $*_{S}$ and obtain a noncombustion wave solution (Figure 4(b)).

3) When $T_{-} \leq T_{i}, T_{S} \leq T_{i}$, then $\beta\left(*_{S}\right)=0, \beta\left(*_{D}\right)=2$, from the condition $A$, we select $*_{s}$ and obtain a noncombustion wave solution (Figure 4(b)).

4) When $T_{-} \leq T_{i}, T_{S}>T_{i}\left(\Rightarrow T_{D}>T_{i}\right)$, then $\beta\left(*_{S}\right)=2, \beta\left(*_{D}\right)=2$, from the condition $B$, we select $*_{D}$ and obtain a combustion wave solution containing a $D F$ or $D T$ (Figure 4(a)).

Subcase 2.1.2 When $u_{-}-u_{+} \geq \sqrt{-\frac{p_{-}}{\rho_{-}}}+\sqrt{-\frac{p_{+}}{\rho_{+}}}$.

In this subcase, we can only consider the delta shock wave solution $S_{\delta}$ connecting the state $(-)$ and $(+)$ which is expressed as follows:

$$
\rho=\rho_{0}+\omega(t) \delta(x-x(t)), \rho_{0}= \begin{cases}\rho_{-}, & x<x(t), \\ \rho_{+}, & x>x(t),\end{cases}
$$




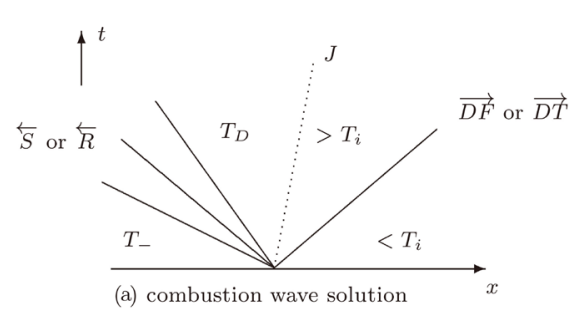

Figure 4. The discussions in Subcase 2.1.1.

$$
\begin{aligned}
& u(x, t)= \begin{cases}u_{-}, & x<x(t), \\
u_{\delta}, & x=x(t), \\
u_{+}, & x>x(t),\end{cases} \\
& p(x, t)= \begin{cases}p_{-}, & x<x(t), \\
0, & x=x(t), \\
p_{+}, & x>x(t),\end{cases}
\end{aligned}
$$

when $\rho_{-} \neq \rho_{+}$,

$$
\begin{gathered}
\omega(t)=\sqrt{\rho_{-} \rho_{+}\left(u_{+}-u_{-}\right)^{2}-\left(\rho_{+}-\rho_{-}\right)\left(p_{+}-p_{-}\right)} t, \\
u_{\delta}=\frac{\rho_{+} u_{+}-\rho_{-} u_{-}+\frac{\mathrm{d} \omega(t)}{\mathrm{d} t}}{\rho_{+}-\rho_{-}},
\end{gathered}
$$

when $\rho_{-}=\rho_{+}$,

$$
\begin{gathered}
\omega(t)=\left(\rho_{-} u_{-}-\rho_{+} u_{+}\right) t, \\
u_{\delta}=\frac{1}{2}\left(u_{-}+u_{+}\right) .
\end{gathered}
$$

Case 2.2. $q_{-}>0, q_{+}>0$ and the gas on both sides are unburnt.

For this case, the backward wave curve is

$\bar{W}(-)=\bar{W}_{S}(-) \cup S_{\delta}(-) \cup \overline{D T}(-) \cup \overline{D F}(-)$, and the forward wave curve is $\vec{W}(+)=\vec{W}_{S}(+) \cup S_{\delta}(+) \cup \overrightarrow{D T}(+) \cup \overrightarrow{D F}(+)$ (Figure 5).

Subcase 2.2.1 When $u_{-}-u_{+}<\sqrt{-\frac{p_{-}}{\rho_{-}}}+\sqrt{-\frac{p_{+}}{\rho_{+}}}$.

If the intersection point of $\bar{W}(-)$ and $\vec{W}(+)$ is unique, the solution of the Riemann problem is also unique; otherwise, there are at most four intersection points of $\bar{W}(-)$ and $\vec{W}(+)$.

We should select the unique Riemann solution from the four solutions under the global entropy conditions (Figure 6).

Since $\beta=0$ for Case (i) and $\beta=2$ for the other three cases, we know from the global entropy conditions that we should select the intersection point 1 and obtain a noncombustion wave solution $\bar{S}$ or $\bar{R}+\vec{S}$ or $\vec{R}$.

Subcase 2.2.2 When $u_{-}-u_{+} \geq \sqrt{-\frac{p_{-}}{\rho_{-}}}+\sqrt{-\frac{p_{+}}{\rho_{+}}}$. There are two possibilities: one is the delta shock wave solution, the other is the $\overline{D T}+\overrightarrow{D T}$. According to the condition A of the global entropy conditions, we choose the delta shock wave solution which is the noncombustion wave solution. 
$\uparrow p$

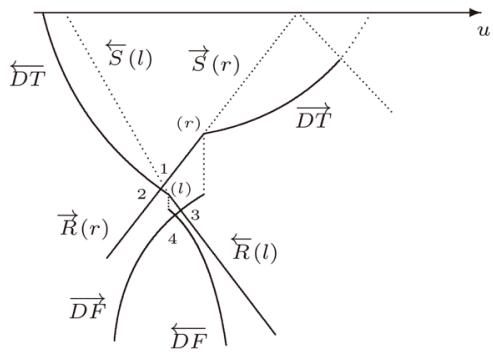

Figure 5. The wave curves in Case 2.2.

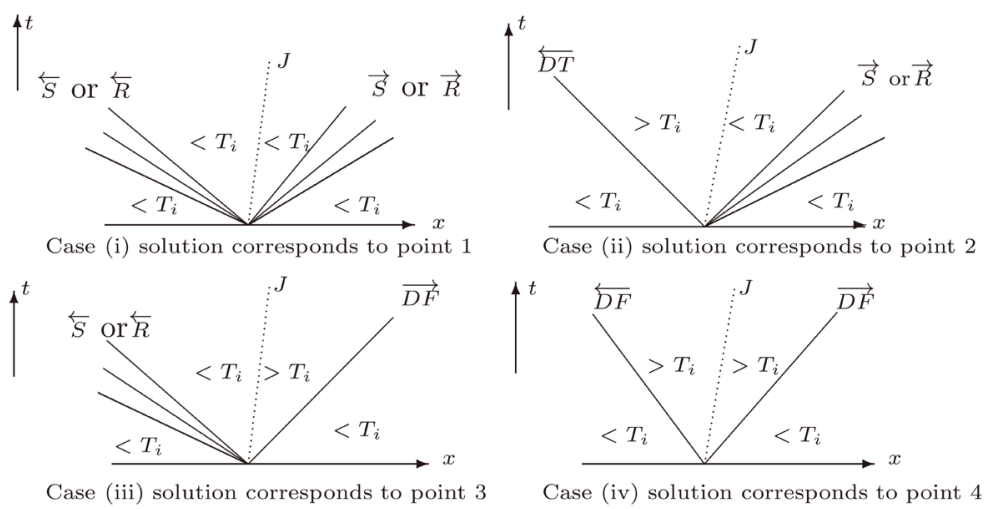

Figure 6. The discussions in Subcase 2.2.1.

Theorem 2.1. Under the global entropy conditions, we construct the unique solution for the Riemann problem (1) with the inial data (4).

\section{Solutions of the Generalized Riemann Problem}

Now we investigate the solutions for the initial value problem (1) and (6) in a neighborhood of the origin $(t>0)$ on the $(x, t)$ plane. From the results in [15], the classical solution $\left(\tau_{l}, u_{l}, p_{l}, q_{l}\right)(x, t) \quad\left(\left(\tau_{r}, u_{r}, p_{r}, q_{r}\right)(x, t)\right)$ can be defined in a strip domain $D_{l}\left(D_{r}\right)$ for a local time. The right boundary of $D_{l}$ has characteristic $O A: x=\lambda_{-} t$, and the left boundary of $D_{r}$ has characteristic $O B: x=\lambda_{+} t$ (Figure 7).

According to the different cases of the corresponding Riemann solutions of (1) and (4), we construct the solutions under the global entropy conditions in [12] case by case for (1) and (6). For simplicity, we only consider some interesting phenomena. For the other cases, similar discussions can be carried out. For simplicity, we use the same symbols after perturbation since there is no any confusion.

Case 1. Assume that $q_{-}=0, q_{+}>0$ and there are three intersection points of $\bar{W}(l)$ and $\vec{W}_{S}(r)$ (Figure 3 ). The corresponding Riemann problem may have a non-combustion solution or a combustion wave solution.

Subcase 1.1. The solution of the corresponding Riemann problem is the noncombustion wave solution. 


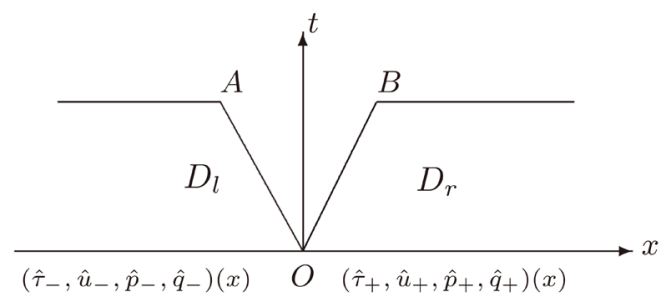

Figure 7. The discussed local region.

Subcase 1.1.1 When $u_{-}-u_{+}<\sqrt{-\frac{p_{-}}{\rho_{-}}}+\sqrt{-\frac{p_{+}}{\rho_{+}}}$. After perturbation we still have $u_{-}-u_{+}<\sqrt{-\frac{p_{-}}{\rho_{-}}}+\sqrt{-\frac{p_{+}}{\rho_{+}}}$and discuss as follows.

Subcase 1.1.1.1 $T_{-} \leq T_{i}, T_{S} \leq T_{i}$ (Figure 4(b)).

After perturbation we obtain $T_{-}>T_{i}$, or $T_{-} \leq T_{i}, T_{D} \leq T_{i}$ or $T_{D}>T_{i}$, $T_{S} \leq T_{i}$ or $T_{S}>T_{i}$, and construct the perturbed solution as follows.

If $T_{-}>T_{i}, T_{D} \leq T_{i}$, then $\beta\left(*_{S}\right)=1, \beta\left(*_{D}\right)=3$, from the condition A we obtain a perturbed Riemann solution which is a non-combusiton wave solution.

If $T_{-}>T_{i}, T_{D}>T_{i}$, then $\beta\left(*_{s}\right)=1, \beta\left(*_{D}\right)=1$, from the condition $\mathrm{B}$ we obtain a perturbed Riemann solution which is a combusiton wave solution.

If $T_{-} \leq T_{i}, T_{S} \leq T_{i}$, then $\beta\left(*_{S}\right)=0, \beta\left(*_{D}\right)=2$, from the condition A we obtain a perturbed Riemann solution which is a non-combusiton wave solution.

If $T_{-} \leq T_{i}, T_{S}>T_{i}$, then $\beta\left(*_{S}\right)=2, \beta\left(*_{D}\right)=2$, from the condition $\mathrm{B}$ we obtain a perturbed Riemann solution which is a combusiton wave solution.

Subcase 1.1.1.2 $T_{-}>T_{i}, T_{D} \leq T_{i}\left(\Rightarrow T_{S} \leq T_{i}\right)$ (Figure 4(b)).

After perturbation we obtain $T_{-}>T_{i}, T_{D} \leq T_{i}$ or $T_{D}>T_{i}, T_{S} \leq T_{i}$ or $T_{S}>T_{i}$, and construct the perturbed solution as follows.

If $T_{-}>T_{i}, T_{D} \leq T_{i}$, then $\beta\left(*_{S}\right)=1, \beta\left(*_{D}\right)=3$, from the condition A we obtain a perturbed Riemann solution which is a non-combusiton wave solution.

If $T_{-}>T_{i}, T_{D}>T_{i}$, then $\beta\left(*_{S}\right)=1, \beta\left(*_{D}\right)=1$, from the condition $\mathrm{B}$ we obtain a perturbed Riemann solution which is a combusiton wave solution.

Subcase 1.1.2 When $u_{-}-u_{+} \geq \sqrt{-\frac{p_{-}}{\rho_{-}}}+\sqrt{-\frac{p_{+}}{\rho_{+}}}$. In this case, the corresponding Riemann solution is a delta shock wave solution. After perturbation we have $u_{-}-u_{+}<\sqrt{-\frac{p_{-}}{\rho_{-}}}+\sqrt{-\frac{p_{+}}{\rho_{+}}}$or $u_{-}-u_{+} \geq \sqrt{-\frac{p_{-}}{\rho_{-}}}+\sqrt{-\frac{p_{+}}{\rho_{+}}}$and discuss as follows. When $u_{-}-u_{+}<\sqrt{-\frac{p_{-}}{\rho_{-}}}+\sqrt{-\frac{p_{+}}{\rho_{+}}}$, it follows that:

If $T_{-}>T_{i}, T_{D} \leq T_{i}$, then $\beta\left(*_{S}\right)=1, \beta\left(*_{D}\right)=3$, from the condition A we obtain a perturbed Riemann solution which is a non-combusiton wave solution;

If $T_{-}>T_{i}, T_{D}>T_{i}$, then $\beta\left(*_{S}\right)=1, \beta\left(*_{D}\right)=1$, from the condition $\mathrm{B}$ we obtain a perturbed Riemann solution which is a combusiton wave solution;

If $T_{-} \leq T_{i}, T_{S} \leq T_{i}$, then $\beta\left(*_{S}\right)=0, \beta\left(*_{D}\right)=2$, from the condition A we obtain a noncombusiton wave solution; 
If $T_{-} \leq T_{i}, T_{S}>T_{i}$, then $\beta\left(*_{S}\right)=2, \beta\left(*_{D}\right)=2$, from the condition B we obtain a perturbed Riemann solution which is a combusiton wave solution.

When $u_{-}-u_{+} \geq \sqrt{-\frac{p_{-}}{\rho_{-}}}+\sqrt{-\frac{p_{+}}{\rho_{+}}}$, it follows that we obtain the perturbed solution is still a delta shock wave solution.

Subcase 1.2. The solution of the corresponding Riemann problem is the combustion wave solution.

When $u_{-}-u_{+}<\sqrt{-\frac{p_{-}}{\rho_{-}}}+\sqrt{-\frac{p_{+}}{\rho_{+}}}$. After perturbation we still have $u_{-}-u_{+}<\sqrt{-\frac{p_{-}}{\rho_{-}}}+\sqrt{-\frac{p_{+}}{\rho_{+}}}$and discuss as follows.

Subcase 1.2.1 $T_{-}>T_{i}, T_{D}>T_{i} \quad$ (Figure 4(a)).

After perturbation we obtain $T_{-}>T_{i}, T_{D}>T_{i}, T_{S} \leq T_{i}$ or $T_{S}>T_{i}$, and it follows that $\beta\left(*_{S}\right)=1, \beta\left(*_{D}\right)=1$, from the condition $\mathrm{B}$ we obtain the perturbed solution is a combusiton wave solution.

Subcase 1.2.2 $T_{-} \leq T_{i}, T_{D}>T_{i}, T_{S}>T_{i}$ (Figure 4(a)).

After perturbation we obtain $T_{-}>T_{i}$, or $T_{-} \leq T_{i}, T_{D}>T_{i}, T_{S}>T_{i}$, and it follows that:

If $T_{-}>T_{i}, T_{D}>T_{i}, T_{S}>T_{i}$, we have $\beta\left(*_{S}\right)=1, \beta\left(*_{D}\right)=1$, from the condition $\mathrm{B}$ we obtain the perturbed solution is a combusiton wave solution;

If $T_{-} \leq T_{i}, T_{D}>T_{i}, T_{S}>T_{i}$, we have $\beta\left(*_{S}\right)=2, \beta\left(*_{D}\right)=2$, from the condition $\mathrm{B}$ we obtain the perturbed solution is a combusiton wave solution.

Case 2. Assume that $q_{-}>0, q_{+}>0$ and there are four intersection points of $\bar{W}(l)$ and $\vec{W}_{S}(r)$ (Figure 5). And the corresponding Riemann solution for this case is a noncombustion solution which is $\bar{S}$ or $\overleftarrow{R}+\vec{S}$ or $\vec{R}$, or a delta shock wave solution.

Subcase 2.1 When $u_{-}-u_{+}<\sqrt{-\frac{p_{-}}{\rho_{-}}}+\sqrt{-\frac{p_{+}}{\rho_{+}}}$, and the corresponding Riemann solution is the noncombustion solution $\overleftarrow{S}$ or $\overleftarrow{R}+\vec{S}$ or $\vec{R}$. After perturbation we still have $u_{-}-u_{+}<\sqrt{-\frac{p_{-}}{\rho_{-}}}+\sqrt{-\frac{p_{+}}{\rho_{+}}}$and obtain a perturbed Riemann solution which is a noncombustion solution $\bar{S}$ or $\bar{R}+\vec{S}$ or $\vec{R}$.

Subcase 2.2 When $u_{-}-u_{+} \geq \sqrt{-\frac{p_{-}}{\rho_{-}}}+\sqrt{-\frac{p_{+}}{\rho_{+}}}$, and the corresponding Riemann solution is the delta shock wave solution. After perturbation we have $u_{-}-u_{+}<\sqrt{-\frac{p_{-}}{\rho_{-}}}+\sqrt{-\frac{p_{+}}{\rho_{+}}}$or $u_{-}-u_{+} \geq \sqrt{-\frac{p_{-}}{\rho_{-}}}+\sqrt{-\frac{p_{+}}{\rho_{+}}}$and discuss as follows.

Subcase 2.2.1 When $u_{-}-u_{+}<\sqrt{-\frac{p_{-}}{\rho_{-}}}+\sqrt{-\frac{p_{+}}{\rho_{+}}}$, we still get a perturbed Riemann solution which is a noncombustion solution $\bar{S}$ or $\bar{R}+\vec{S}$ or $\vec{R}$.

Subcase 2.2.2 When $u_{-}-u_{+} \geq \sqrt{-\frac{p_{-}}{\rho_{-}}}+\sqrt{-\frac{p_{+}}{\rho_{+}}}$, we obtain a perturbed Riemann 
solution which is delta shock wave solution.

\section{Conclusions}

Based on the above analysis, we find that, for most cases, the corresponding Riemann solutions are stable under the global entropy conditions. While for some other cases, a small perturbation on the initial data may lead to essential changes to the corresponding Riemann solutions. The perturbation may make the combustion wave extinguished. For some cases, although the corresponding Riemann solution doesn't contain combustion wave burning happens after perturbation which shows the instability of the unburnt gas. Notice that there are much richer structures of the perturbed Riemann solutions of our combustible model (1) and (6) than that of the conventional combustible gas dynamics model (2) and (6).

Theorem 4.1. Under the global entropy conditions, there exists uniquely solution for the generalized Riemann problem (1) and (6) in a neighborhood of the origin $(t>0)$ on the $(x, t)$ plane.

Notice that the reaction velocity in our CJ model is infinite, which is an idealized hypothesis, while our model is still very important in many applications, we will discuss the initial value problem for the ZND model with finite reaction velocity in our coming works.

\section{Conflicts of Interest}

The author declares no conflicts of interest regarding the publication of this paper.

\section{References}

[1] Courant, R. and Friedrichs, K.O. (1948) Supersonic Flow and Shock Waves. Interscience, New York.

[2] Chaplygin, S. (1904) On Gas Jets. Scientific Memoirs, Moscow University Mathematic Physics, 21, 1-121.

[3] Brenier, Y. (2005) Solutions with Concentration to the Riemann Problem for OneDimensional Chaplygin Gas Equations. Journal of Mathematical Fluid Mechanics, 7, 326-331. https://doi.org/10.1007/s00021-005-0162-x

[4] Guo, L.H., Sheng, W.C. and Zhang, T. (2010) The Two-Dimensional Riemann Problem for Isentropic Chaplygin Gas Dynamics System. Communications on Pure \& Applied Analysis, 9, 431-458. https://doi.org/10.3934/cpaa.2010.9.431

[5] Lai, G., Sheng, W.C. and Zheng, Y.X. (2011) Simple Waves and Pressure Delta Waves for a Chaplygin Gas in Two-Dimensions. Discrete \& Continuous Dynamical Systems, 31, 489-523. https://doi.org/10.3934/dcds.2011.31.489

[6] Serre, D. (2009) Multidimensional Shock Interaction for a Chaplygin Gas. Archive for Rational Mechanics and Analysis, 191, 539-577. https://doi.org/10.1007/s00205-008-0110-Z

[7] Williams, F.A. (1985) Combustion Theory. Benjamin Commings, Menlo Park.

[8] Zhang, T. and Zheng, Y.X. (1989) Riemann Problem for Gas Dynamic Combustion. 
Journal of Differential Equations, 77, 203-230.

https://doi.org/10.1016/0022-0396(89)90142-3

[9] Liu, Y.J. and Sheng, W.C. (2011) Generalized Riemann Problem for Gas Dynamic Combustion. Applied Mathematics and Mechanics, 32, 1079-1090.

https://doi.org/10.1007/s10483-011-1482-6

[10] Tan, D.C. and Zhang, T. (1992) Riemann Problem for the Self-Similar ZND Model in Gas Dynamical Combustion. Journal of Differential Equations, 95, 331-369. https://doi.org/10.1016/0022-0396(92)90035-L

[11] Hsu, C.H. and Lin, S.S. (1997) Some Qualitative Properties of the Riemann Problem in Gas Dynamical Combustion. Journal of Differential Equations, 140, 10-43. https://doi.org/10.1006/jdeq.1997.3304

[12] Liu, Y.J. (2015) Riemann Problem for Chaplygin Gas with Combustion. Applicable Analysis, 94, 1877-1887. https://doi.org/10.1080/00036811.2014.956732

[13] Zhu, L. (2010) Chaplygin Gas Dynamic System in Adiabatic Flow. Master Thesis, Shanghai University, Shanghai.

[14] Sheng, W.C. and Zhang, T. (1999) The Riemann Problem for the Transportation Equations in Gas Dynamics. Memoirs of the American Mathematical Society, 137, 1-77. https://doi.org/10.1090/memo/0654

[15] Li, T.T. and Yu, W.C. (1985) Boundary Value Problem for Quasilinear Hyperbolic Systems. Duke University Mathematics Series V, Durham. 